\title{
ARTICLE
}

\section{T-448, a specific inhibitor of LSD1 enzyme activity, improves learning function without causing thrombocytopenia in mice}

Satoru Matsuda ${ }^{1}$, Rina Baba ${ }^{1}$, Hideyuki Oki ${ }^{2}$, Shinji Morimoto ${ }^{1}$, Masashi Toyofuku' ${ }^{1}$, Shigeru Igaki ${ }^{2}$, Yusuke Kamada (D) ${ }^{2}$, Shinji Iwasaki ${ }^{3}$, Kota Matsumiya ${ }^{3}$, Ryosuke Hibino ${ }^{1}$, Hiroko Kamada ${ }^{1}$, Takeshi Hirakawa ${ }^{1}$, Misa Iwatani ${ }^{2}$, Ken Tsuchida ${ }^{4}$, Ryujiro Hara ${ }^{1}$, Mitsuhiro Ito ${ }^{1}$ and Haruhide Kimura ${ }^{1}$

Dysregulation of histone $\mathrm{H} 3$ lysine 4 (H3K4) methylation has been implicated in the pathogenesis of several neurodevelopmental disorders. Targeting lysine-specific demethylase 1 (LSD1), an H3K4 demethylase, is therefore a promising approach to treat these disorders. However, LSD1 forms complexes with cofactors including growth factor independent 1B (GFI1B), a critical regulator of hematopoietic differentiation. Known tranylcypromine-based irreversible LSD1 inhibitors bind to coenzyme flavin adenine dinucleotide (FAD) and disrupt the LSD1-GFI1B complex, which is associated with hematotoxicity such as thrombocytopenia, representing a major hurdle in the development of LSD1 inhibitors as therapeutic agents. To discover LSD1 inhibitors with potent epigenetic modulation and lower risk of hematotoxicity, we screened small molecules that enhance H3K4 methylation by the inhibition of LSD1 enzyme activity in primary cultured rat neurons but have little impact on LSD1-GFI1B complex in human TF-1a erythroblasts. Here we report the discovery of a specific inhibitor of LSD1 enzyme activity, T-448 (3-((1S,2R)-2-(cyclobutylamino) cyclopropyl)- $\mathrm{N}$-(5-methyl-1,3,4-thiadiazol-2-yl)benzamide fumarate). T-448 has minimal impact on the LSD1-GFI1B complex and a superior hematological safety profile in mice via the generation of a compact formyl-FAD adduct. T-448 increased brain H3K4 methylation and partially restored learning function in mice with NMDA receptor hypofunction. T-448-type LSD1 inhibitors with improved safety profiles may provide unique therapeutic approaches for central nervous system disorders associated with epigenetic dysregulation.

Neuropsychopharmacology (2019) 44:1505-1512; https://doi.org/10.1038/s41386-018-0300-9

\section{INTRODUCTION}

Epigenetic control of gene transcription by histone $\mathrm{H} 3$ lysine 4 (H3K4) methylation is critically involved in neural functions such as learning and memory through the regulation of synaptic plasticity [1-3]. Upregulation of H3K4 methylation levels allows active transcription of genes via recruitment of chromatin remodeling factors. Reduced methylation of H3K4, and the consequent reduction of gene transcription, have been implicated in the pathogenesis of various psychiatric and neurological disorders, such as schizophrenia $[4,5]$, autism spectrum disorders (ASD) $[6$, 7], intellectual disability disorders [8], and Huntington's disease [9]. Therefore, treatments which restore normal levels of H3K4 methylation may be a promising approach to treat disorders associated with this type of epigenetic dysfunction.

Lysine-specific demethylase 1 (LSD1, also known as KDM1A) is an enzyme that decreases methylation levels, predominantly at H3K4 [10]. Interrestingly, LSD1 only demethylates histone H3 lysine 9 (H3K9) when the molecule forms a complex with an androgen receptor [11]. Non-histone proteins such as DNMT1 [12] are also known LSD1 substrates. At H3K4, LSD1 catalyzes the oxidative removal of methyl groups from mono- and dimethylated $\mathrm{H} 3 \mathrm{~K} 4$ by reducing flavin adenine dinucleotide (FAD) into $\mathrm{FADH}_{2}$ [13-16]. The principal target site of known irreversible LSD1 inhibitors is FAD, located within the active site cavity of LSD1 [17]. This appears to be the most promising approach to discover LSD1 inhibitors, as no allosteric LSD1 inhibitors have been identified.

LSD1 interacts with multiple cofactor proteins such as growth factor independent 1B (GFI1B), a Snail/Gfi1 (SNAG) domain family protein in the vicinity of FAD binding site $[18,19]$. GFI1B regulates the hematopoietic differentiation of erythroid and megakaryocytic cells by repressing the transcription of most of its target genes, including growth factor independent 1 (GFI1) [20,21]. Depletion of LSD1 by knockdown in mice derepressed the expression of the genes controlled by GFI1B $[19,22]$ and attenuated the production of peripheral blood cells by perturbing hematopoietic differentiation. Moreover, known irreversible LSD1 inhibitors with a tranylcypromine structure that produce FAD-adducts have shown in vivo hematological toxicities, such as thrombocytopenia [23-25], probably through disruption of the interaction between LSD1 and cofactors [26, 27]. Reversible inhibitors have not been reported to form FAD-adducts. Hematological toxicity caused by disruption of the LSD1-complex may pose a major hurdle in the development of LSD1 inhibitors as therapeutic agents.

\footnotetext{
${ }^{1}$ Neuroscience Drug Discovery Unit, Research, Takeda Pharmaceutical Company Limited, 26-1, Muraoka-Higashi 2-chome, Fujisawa, Kanagawa 251-8555, Japan; ${ }^{2}$ Biomolecular

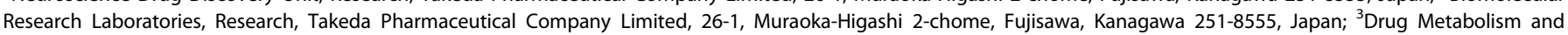

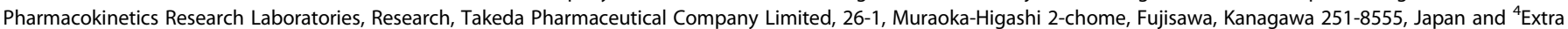
Value Generation Drug Discovery Unit, Research, Takeda Pharmaceutical Company Limited, 26-1, Muraoka-Higashi 2-chome, Fujisawa, Kanagawa 251-8555, Japan Correspondence: Haruhide Kimura (haruhide.kimura@takeda.com)
} 
Here, we report the discovery of a novel selective LSD1 inhibitor, 3-((1S,2R)-2-(cyclobutylamino)cyclopropyl)- $\mathrm{N}$-(5-methyl1,3,4-thiadiazol-2-yl)benzamide fumarate (T-448) using an original screening strategy. T-448 irreversibly inhibited the enzyme activity of LSD1 through the generation of a compact formyl-FAD adduct. Formation of the formyl-FAD adduct had minimal impact on the interaction between LSD1 and GFI1B and as expected, $\mathrm{T}-448$ at the effective dose did not show hematological toxicity in mice. In mice with $N$-methyl-D-aspartate receptor (NMDAR) hypofunction, a preclinical model of schizophrenia and ASD [28-30], T-448 enhanced the levels of H3K4 methylation, increased mRNA expression of neural plasticity-related genes including brain derived neurotrophic factor (Bdnf), and ameliorated learning dysfunction as measured in the water Y-maze test. The evidence we provide here suggests that T-448-type LSD1 inhibitors may provide a novel therapeutic opportunity for central nervous system (CNS) diseases associated with epigenetic dysregulation.

\section{MATERIALS AND METHODS}

See supplemental information for detailed methods. The methods of statistical analysis and criteria of significance are indicated in each Figure legend.

\section{Animals}

All experiments were approved by the Institutional Animal Care and Use Committee of Shonan Research Center, Takeda Pharmaceutical Company Limited. Male Jcl:ICR mice were obtained from CLEA Japan, Inc. Primary neuronal culture was prepared from Crl: $\mathrm{CD}(\mathrm{SD})$ rats at embryonic day 19 purchased from Charles River Laboratories Japan, Inc. [31].

\section{TF-1a culture}

TF-1a was purchased from the American Tissue Culture Collection. Cell culture, viability assay, and immunoprecipitation were performed as previously described [23] with minor modifications.

\section{H3K4me2 ChIP studies}

ChIP was performed by using ChIP-IT Express Enzymatic (Active Motif, \#53009) or OneDay ChIP Kit (Nippon Gene, \#313-80461) and H3K4me2 antibody (Millipore, \#07-030) with a slight modification to the manufacturer's instruction. Sequencing was performed by Takara Bio Inc. (HiSeq2000).

mRNA expression studies

mRNA expression was quantified by using FastLane kit (QIAGEN) or RNeasy Plus Kit (QIAGEN) and TaqMan reagents (Eurogentec) following the manufacturer's instruction. Microarray study was performed by Takara Bio Inc. (SurePrint G3 Rat Gene Expression $8 \times 60 \mathrm{~K})$.

\section{SPR analysis}

Experiments for binding to recombinant human LSD1 protein were carried out using Biacore 4000 instrument equipped with Sensor chip CM5 (GE Healthcare).

Structural analyses

Crystals were obtained using the sitting-drop vapor-diffusion method. Diffraction data for each complex crystal were collected at $100 \mathrm{~K}$ at the Advanced Light Source beamline 5.0.3 or 5.0.2 (Berkeley). ESI-triple quadrupole mass spectrometry was used for the analysis of FAD-adducts.

\section{Ex vivo LSD1 enzyme assay}

LSD1 protein in mouse tissue homogenates was immunoprecipitated by using anti-LSD1 antibody (Cell Signaling Technology, \#2139) and Protein G Sepharose 4FF, according to the manufacturer's instructions. Histone demethylase activity in immunoprecipitates was measured by $\mathrm{HTRF}^{\mathrm{TM}}$ detection system.

Cognition test

Learning function was assessed by water Y-maze test using NR1hypo $\left(\mathrm{Nr}^{\text {neo- }-}-\right)$ mice and their wild-type littermates $[28,29]$.

\section{RESULTS}

Discovery of a novel LSD1 inhibitor, T-448

To discover LSD1 inhibitors with potent epigenetic modulation and lower risk of hematological toxicity, we set the following three criteria for the selection of lead compounds: (1) selective inhibition of the enzyme activity of purified human recombinant LSD1, (2) increase in $\mathrm{H} 3 \mathrm{~K} 4$ methylation levels and the consequent induction of gene transcription in primary cultured rat neurons, and (3) minimal impact on the transcription of GFI1 gene in human TF-1a erythroblast cell line $[19,22]$. We also assessed the dissociation of the LSD1-GFI1B complex as a representative mechanism that controls GFI1 mRNA expression in TF-1a cells [20, 21].

Chromatin immunoprecipitation (ChIP) is a method to quantitatively evaluate H3K4 methylation levels at specific genomic regions; however, the high-throughput screening of drugs using ChIP was technically difficult owing to the required amounts of primary cultured neurons and the complicated procedures involved. Therefore, we decided to use the induction of surrogate marker genes for the initial search. Surrogate marker genes were identified using T-711 (Fig. 1a), a racemic irreversible-type LSD1 inhibitor with specificity constant $k_{\text {inact }} / K_{\text {l }}$ of $3.8 \times 10^{5} \pm 1.9 \times 10^{4}$ $\left(\mathrm{s}^{-1} \mathrm{M}^{-1}\right)$ (Fig. S1) [32]. The genes with increased levels of H3K4 methylation and mRNA expression after treatment with T-711 in primary cultured rat neurons represented candidates for surrogate marker genes. The effects of T-711 $(1 \mu \mathrm{M})$ on H3K4 di-methylation (H3K4me2) in primary cultured rat neurons were studied by ChIPsequencing (ChIP-seq) using an antibody against H3K4me2 in a genome-wide manner. The sequence reads were mapped to rat genomes (rn4), and H3K4me2 peaks were called by model-based analysis of ChIP-seq (MACS) algorithm. Among them, peaks located within genes or promoter regions were annotated to the gene with the nearest transcription start sites (TSSs). As a result, we identified 136 genes with more than 4-fold increases in H3K4me2 levels after treatment with T-711 (Fig. 1b). The changes in mRNA expression induced by T-711 $(1 \mu \mathrm{M})$ in primary cultured rat neurons were analyzed through a microarray study; 55 genes showed more than 2-fold increased expression (Fig. 1b). Among the 10 genes identified by both H3K4me2 ChIP-seq and microarray analyses, Ucp2 mRNA showed the most robust fold induction (Table S1). H3K4me2 ChIP-quantitative PCR (qPCR) and RT-qPCR analyses revealed that T-711 significantly increased the H3K4me2 level at the upstream region ( -239 base pairs from the TSS) of the Ucp2 gene, and increased mRNA expression of the Ucp2 gene in a concentration-dependent manner in primary cultured rat neurons (Fig. 1c). Therefore, we decided to utilize the Ucp2 mRNA levels in primary cultured rat neurons as a surrogate marker to guide discovery of LSD1 inhibitors.

GFI1 regulates hematopoietic differentiation, and GFI1 mRNA transcription is controlled by an LSD1-complex including GFI1B in hematopoietic lineage cells $[19,21,22]$. Therefore, we decided to use the induction of GFI1 mRNA expression in TF-1a erythroblast cell lines as a screening index of hematological toxicity. RT-qPCR analysis confirmed that T-711 significantly increased the GFI1 mRNA expression in a concentration-dependent manner in TF-1a cells (Fig. 1d). T-711 reduced the viability of TF-1a cells at the concentrations where it induced the GFI1 mRNA expression (Fig. 1d).

We initially screened our tranylcypromine-focused compound library (663 compounds) and discovered 440 compounds with inhibitory activity against human recombinant LSD1 $(<100 \mathrm{nM}$ 
a

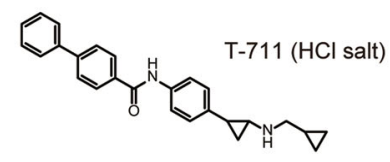

b

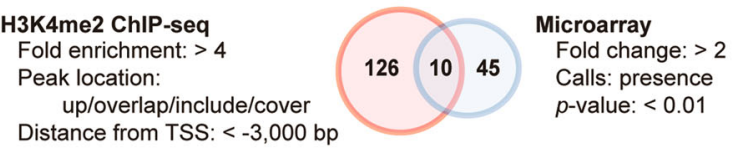

C
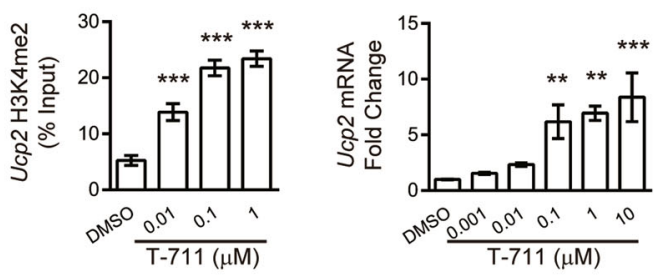

d
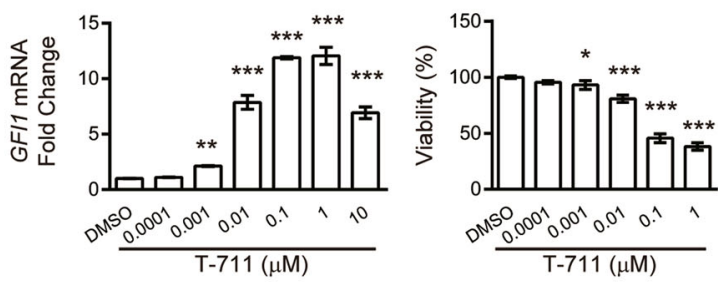

e

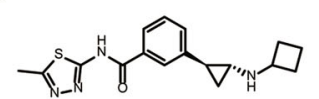

T-448 (fumarate salt)

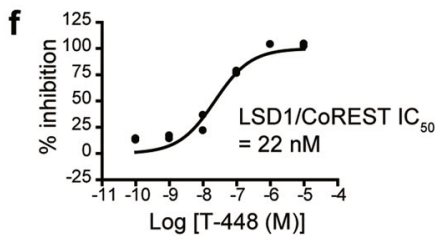

g
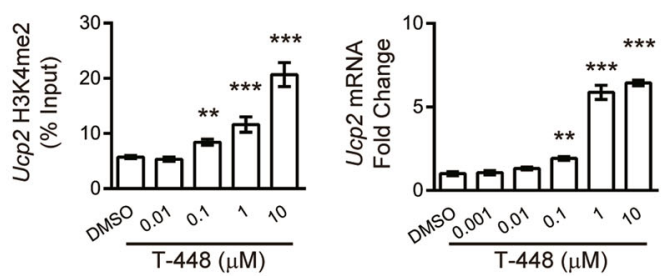

h
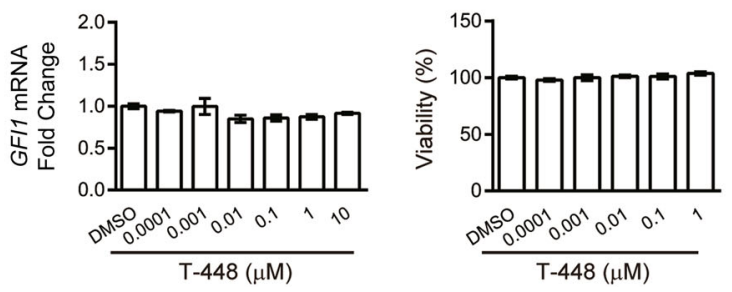

$\left.I C_{50}\right)$. Next, we selected compounds that increased Ucp2 mRNA expression in primary cultured rat neurons in a concentrationdependent manner (criteria: $>2$-fold change at $1 \mu \mathrm{M}$ ) without affecting GFI1 mRNA expression in TF-1a cells (criteria: <2-fold change at $10 \mu \mathrm{M})$. Then, two compounds were chosen because of the good selectivity against monoamine oxidase (MAO) A and B inhibition and their drug-likeness. Finally, T-448 (Fig. 1e) was selected owing to its better brain penetration profile. T-448 inhibited H3K4 demethylase activity of human recombinant LSD1 with an $\mathrm{IC}_{50}$ value of $22 \mathrm{nM}$ in an irreversible manner: $k_{\text {inact }} / K_{l}$ value of $1.7 \times 10^{4} \pm 2.6 \times 10^{3}\left(\mathrm{~s}^{-1} \mathrm{M}^{-1}\right)$ (Fig. If and Fig. S1). T-448 showed more than 4,500-fold selectivity over MAOA/B: the FADdependent enzymes with high amino acid sequence homology to
Fig. 1 T-448 inhibited enzyme activity of LSD1 and increased H3K4me2 in neurons but did not impact the GFI1 mRNA levels in TF1 a erythroblast cells. a Chemical structure of T-711. b Venn diagram showing the numbers of genes with increased $\mathrm{H} 3 \mathrm{~K} 4 \mathrm{me} 2$ and mRNA expression by $\mathrm{T}-711$ treatment $(1 \mu \mathrm{M})$ for 3 days in primary cultured rat neurons. Detailed information of overlapped 10 genes is shown in Table S1. c Effects of 3-day treatment with T-711 on Ucp2 gene H3K4me2 and Ucp2 mRNA levels in primary cultured rat neurons. Graphs indicate mean \pm SEM. $n=4$ (DMSO group in H3K4me2 assay) or 3 (other groups). One-tailed parametric Williams' test vs DMSOtreatment group, ${ }^{* *} p<0.005,{ }^{* * *} p<0.0005$. d Effects of T-711 on GFI1 mRNA levels (1-day treatment) and viability (3-day treatment) in TF-1a erythroblast cells. Graphs indicate mean \pm SEM. $n=18$ (DMSO group in GFI1 assay), $n=15$ (DMSO group in viability assay), or 3 (compound treatment groups). One-tailed (GFI1 assay) and twotailed (viability assay) parametric Williams' test vs DMSO-treatment group, ${ }^{*} p<0.025,{ }^{* *} p<0.005,{ }^{* * *} p<0.0005$. e Chemical structure of T-448. $\mathbf{f}$ Inhibition of LSD1 enzyme activity by T-448. The IC $\mathrm{C}_{50}$ value was determined as $22 \mathrm{nM}$ (95\% confidence interval (Cl): $13-40 \mathrm{nM}$ ). $n=2$. $g$ Effects of 3-day treatment with T-448 on Ucp2 gene H3K4me2 and Ucp2 mRNA levels in primary cultured rat neurons. Graphs indicate mean \pm SEM. $n=3$. One-tailed parametric Williams' test vs DMSO-treatment group, ${ }^{* *} p<0.005,{ }^{* * *} p<0.0005$. h Effects of T-448 on GFI1 mRNA levels (1-day treatment) and viability (3-day treatment) in TF-1a erythroblast cells. Graphs indicate mean \pm SEM. $n=18$ (DMSO group in GFI1 assay), $n=15$ (DMSO group in viability assay), or 3 (compound treatment groups). No significance by onetailed (GFI1 assay) and two-tailed (viability assay) parametric Williams' test vs DMSO-treatment group

LSD1 (Fig. S2) [33]. T-448 at $0.1 \mu \mathrm{M}$ or higher enhanced both the $\mathrm{H} 3 \mathrm{~K} 4 \mathrm{me} 2$ level at the upstream region of Ucp2 gene and the mRNA expression level of Ucp2 gene in primary cultured rat neurons (Fig. 1g). On the other hand, T-448 at up to $10 \mu \mathrm{M}$ did not increase GFI1 mRNA expression and, at up to $1 \mu \mathrm{M}$, did not affect the viability of TF-1a cells (Fig. $1 \mathrm{~h}$ ).

T-448 inhibits LSD1 enzyme activity with minimal impact on LSD1GFI1B complex

To investigate the mechanism of action underlying the lack of GFI1 mRNA induction by T-448, we assessed the effects of T-448 on the T-711-induced GFI1 mRNA expression in TF-1a cells. TF-1a cells were pre-incubated with DMSO or T-448 $(1 \mu \mathrm{M})$ for $6 \mathrm{~h}$, and then stimulated with various concentrations of T-711 for $24 \mathrm{~h}$. T-448 pre-treatment significantly weakened the T-711-induced GFI1 mRNA expression (Fig. 2a). Thus, T-711 and T-448 may share the same binding site on LSD1.

To understand the impact of T-448 on the LSD1-complex, we evaluated its effects on the interaction between LSD1 and GFI1B in TF-1a cells by immunoprecipitation (IP) analysis, using T-711 as a control. As expected, T-711 robustly reduced the interaction between LSD1 and GFI1B (Fig. 2b, lanes 9, 12, and Fig. S3), while $\mathrm{T}-448(1$ and $10 \mu \mathrm{M})$ did not disrupt the interaction (Fig. 2b, lanes 9-11). Next, TF-1a cells were pre-incubated with DMSO or T-448 $(1$ and $10 \mu \mathrm{M})$ for $6 \mathrm{~h}$, and then treated with T-711 $(1 \mu \mathrm{M})$ for $17 \mathrm{~h}$. As a result, T-448 pre-treatment inhibited the T-711-induced dissociation between LSD1 and GFI1B (Fig. 2b, lanes 12-14). These data further supported the idea that both compounds occupy the same binding site on LSD1, and that T-448 has a lower effect on the interaction between LSD1 and GFI1B than T-711 in TF-1a cells.

The effects of LSD1 inhibitors on the binding between LSD1 and GFI1B were also investigated by surface plasmon resonance (SPR) biosensing assay (Fig. S4). Recombinant human LSD1 was immobilized on a sensor chip, and was then treated with T-711 $(10 \mu \mathrm{M})$ or $\mathrm{T}-448(10 \mu \mathrm{M})$. At both $7.5 \mathrm{~min}$ and $10 \mathrm{~h}$ after $\mathrm{T}-711$ treatment, the binding of GFI1B $(1 \mu \mathrm{M})$ to the LSD1 was attenuated (Fig. 2c, d). T-448-treatment reduced the binding between GFI1B and LSD1 at $7.5 \mathrm{~min}$ after the reaction. At $10 \mathrm{~h}$ 
a

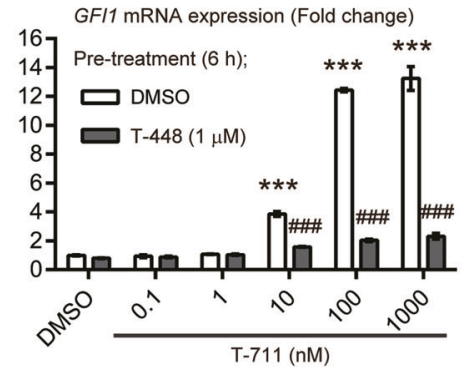

C GFI1B binding at $7.5 \mathrm{~min}$ after LSD1i treatment

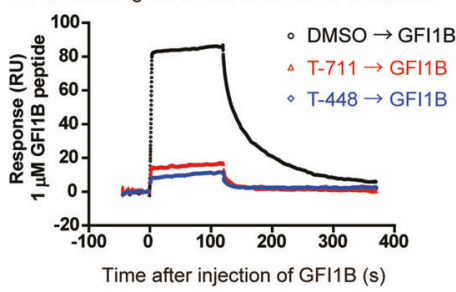

b

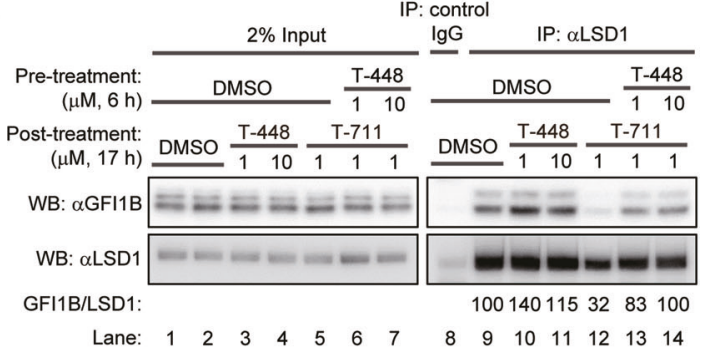

d GFI1B binding at $10 \mathrm{~h}$ after LSD1i treatment

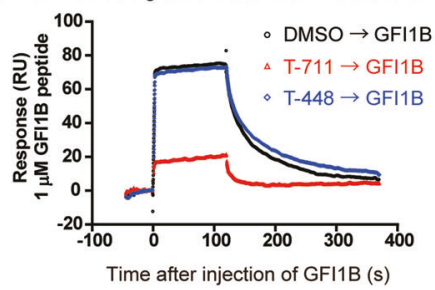

Fig. 2 T-448 showed minimal impact on the LSD1-GFI1B complex. a Effects of pre-treatment with T-448 on T-711-induced GF/1 mRNA expression in TF-1a cells. Graphs indicate mean \pm SEM. $n=6$ (DMSO-treatment group) or 3 (T-711 treatment group). Concentration-dependent effects of T-711 were statistically analyzed by one-tailed parametric Williams' test, ${ }^{* *} p<0.0005$. Effects of T-448 pre-treatment on the inhibition of T-711 (10-1,000 nM)-induced GFI1 mRNA expression were statistically analyzed by Student's t-test with Bonferroni correction, $\# \# \# p<0.00033$. $n=3$. b IP analysis of the interaction between LSD1 and GFI1B in TF-1a cells. GFI1B/LSD1 values are obtained from the densitometry data of GFI1B bands normalized by LSD1 bands (lane $9=100 \%$ ). c, d SPR analysis to measure the effects of LSD1 inhibitors on the interaction between LSD1 and GFI1B, $7.5 \mathrm{~min}(\mathbf{c})$ and $10 \mathrm{~h}(\mathbf{d})$ after the compound treatment $(10 \mu \mathrm{M})$ on immobilized LSD1

after treatment with T-448, the interaction between GFI1B and LSD1 was recovered to the DMSO-control level (Fig. 2c, d). These results suggested that, differently from T-711, dissociation of the interaction between LSD1 and GFI1B by T-448 is only transient.

Formation of a compact FAD-adduct by T-448 permits LSD1-GFI1B interaction

We next analyzed the structures of coenzyme FAD in recombinant human LSD1 after treatment with LSD1 inhibitors. X-ray crystal analysis revealed that T-367 (Fig. S5), the eutomer of T-711, generated a covalent adduct with FAD in the active site cavity of LSD1 (Fig. 3a, stick model in white). LC/MS analysis was used to determine the molecular weight of FAD or FAD-adducts in recombinant LSD1. The peak with $\mathrm{m} / \mathrm{z} 784$ represented intact FAD. LC/MS analysis of FAD in LSD1 treated by T-367 allowed the detection of a peak with $m / z 1,113$, along with the FAD peak, confirming the formation of a T-367-FAD adduct (Fig. 3b). The Nterminal SNAG domain of GFI1B binds to the active site cavity of LSD1 [19]. The binding structure of GFI1B to LSD1 was simulated from the co-crystal analysis of LSD1 with $\mathrm{N}$-terminal SNAIL1 peptide (PDB ID: 2Y48) [18], because the first 6 residues at the $\mathrm{N}$ terminal are identical among SNAIL1 and GFI1B (peptide sequence: PRSFLV). As shown in Fig. 3a, the superimposed structures indicated a steric hindrance between the T-367-FAD adduct and GFI1B peptide (stick model in yellow). These results suggested that the bulky FAD-adduct generated by T-367 disrupts the interaction between LSD1 and GFI1B.

Interestingly, T-448 produced a reduced form of FAD with a formyl group (Fig. $3 c$ and Fig. S6). The formation of a covalent adduct to FAD supports the irreversible inhibition of LSD1 enzyme activity. LC/MS analysis confirmed that the T-448 treatment generated a peak with $m / z$ 814, which corresponded to the molecular weight of FAD with formyl group, and a peak with $\mathrm{m} / \mathrm{z}$ 784 (FAD) (Fig. 3d). This LC/MS study also allowed the detection of a peak with $\mathrm{m} / \mathrm{z} 1,059$, which corresponded to the molecular weight of a full T-448-FAD adduct (Fig. 3d). The $\mathrm{m} / \mathrm{z} 814$ peak count increased while the $m / z 1,059$ peak count decreased in a time-dependent manner, suggesting that the full T-448-FAD adduct is an intermediate product toward formyl-FAD generation (Fig. S7). The resultant formyl-FAD adduct was compact (Fig. 3c, stick model in white), and seemed to show no steric clash with the GFI1B peptide in the active site of LSD1 (Fig. 3c, stick model in yellow). The LC/MS peak with $\mathrm{m} / \mathrm{z} 814$ was not detected upon T367 treatment (Fig. 3d). These data support the hypothesis that T448 inactivates LSD1 enzyme activity through generating a compact formyl-FAD adduct, and the formation of formyl-FAD adduct has minimal impact on the interaction between LSD1 and GFI1B.

T-448 increases $\mathrm{H} 3 \mathrm{~K} 4$ methylation in the brain without causing hematological side effects

In vivo inhibition of LSD1 enzyme activities by T-711 or T-448 can be accurately determined by an ex vivo LSD1 enzyme assay due to their irreversible nature in LSD1 inhibition. Both T-711 and T-448 showed a similar half-life in PK studies in ICR mice; Tmax values of both compounds was achieved $\sim 1 \mathrm{~h}$ after oral administration in ICR mice, and then plasma concentrations declined in a timedependent manner (Fig. S8). We collected tissues $2 \mathrm{~h}$ after the administration of these compounds and conducted ex vivo LSD1 enzyme assays.

Cortex and hippocampus were selected as representative brain regions that are critical for learning and memory functions. The inhibition of LSD1 enzyme activities by T-711 and T-448 in the brain were examined in ICR mouse hippocampus. Both compounds showed dose-dependent inhibition of LSD1 enzyme activities with full inhibition at $10 \mathrm{mg} / \mathrm{kg}$ in the mouse hippocampus (Fig. 4a). The effects of these compounds on H3K4me2 levels at the upstream region of the Ucp2 gene in the mouse cortex were determined after a 9-day repeated administration at up to $10 \mathrm{mg} / \mathrm{kg}$. Both compounds significantly increased the H3K4me2 levels at all doses tested (Fig. 4b). The effects of these compounds on gene expression were examined in the same experimental paradigm; the significant induction of Ucp2 mRNA was observed after administration of T-711 (significant at $1 \mathrm{mg} / \mathrm{kg} /$ day or higher) and T-448 (significant at $3 \mathrm{mg} / \mathrm{kg} /$ day or higher) in the mouse hippocampus (Fig. 4c). 

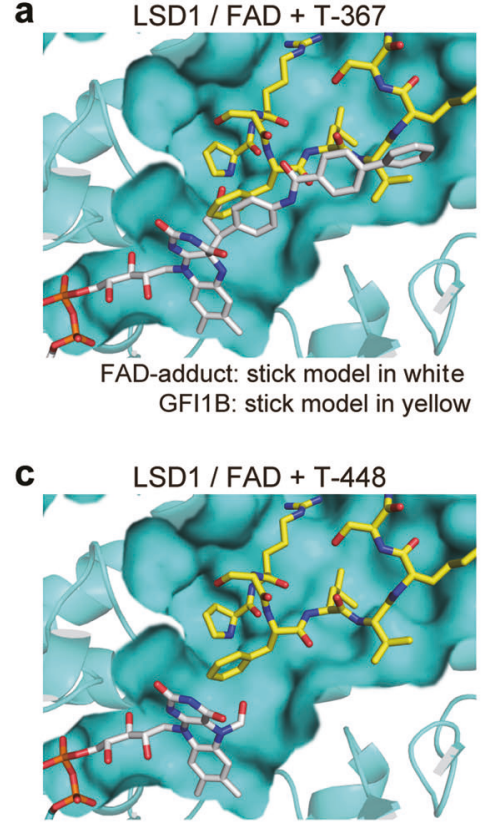

FAD-adduct: stick model in white GFI1B: stick model in yellow
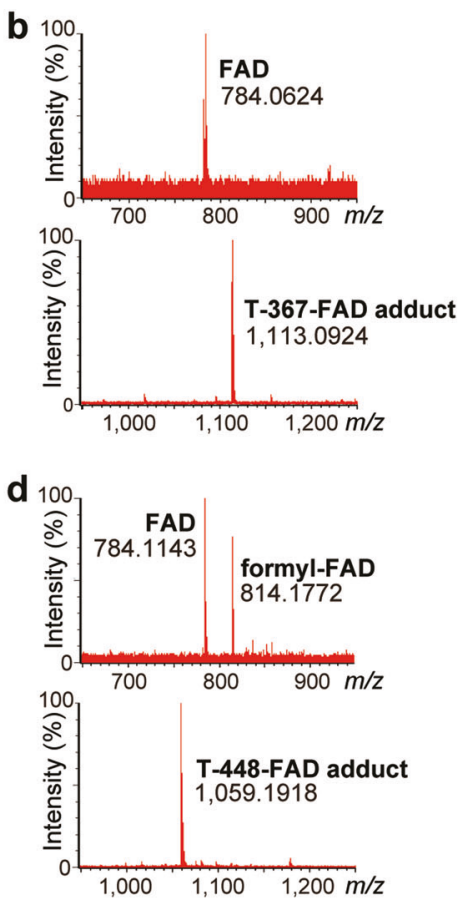

FAD

$[\mathrm{M}-\mathrm{H}]^{-}$Calcd for 784.1493<smiles>Cc1cc2nc3c(=O)[nH]c(=O)nc-3n(C[C@H](O)[C@H](O)[C@H](O)COP(=O)(O)OP(=O)(O)OC[C@H]3O[C@@H](n4cnc5c(N)ncnc54)[C@H](O)[C@@H]3O)c2cc1C</smiles>

formyl-FAD

[M-H] Calcd for 814.1599

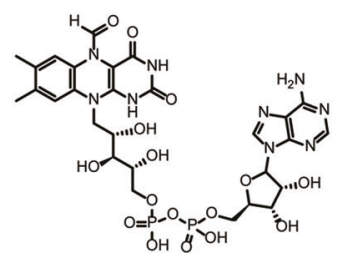

T-367-FAD adduct

[M-H]- Calcd for 1,113.2909

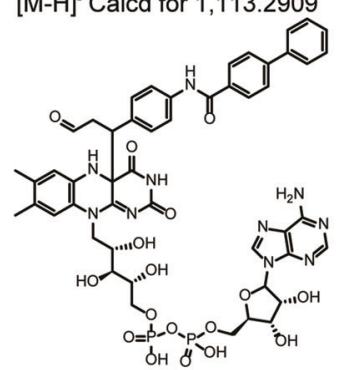

T-448-FAD adduct

[M-H] Calcd for 1,059.2222

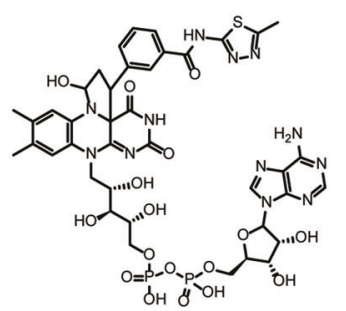

Fig. 3 T-448 produced a compact formyl-FAD adduct in LSD1. a Superimposed structures of T-367-FAD adduct in human LSD1 by crystal analysis (stick model in white) and of N-terminal GFI1B peptide (PRSFLV) in the LSD1-complex by modeling (stick model in yellow). $\mathbf{b}$ LC/MS spectra of FAD and FAD-adducts extracted from human recombinant LSD1 treated with T-367. c Superimposed structures of formylated FAD in LSD1 generated after T-448 treatment by crystal analysis (stick model in white) and N-terminal GFI1B peptide (PRSFLV) in the LSD1-complex by modeling (stick model in yellow). $\mathbf{d}$ LC/MS spectra of FAD and FAD-adducts extracted from human recombinant LSD1 treated with T-448
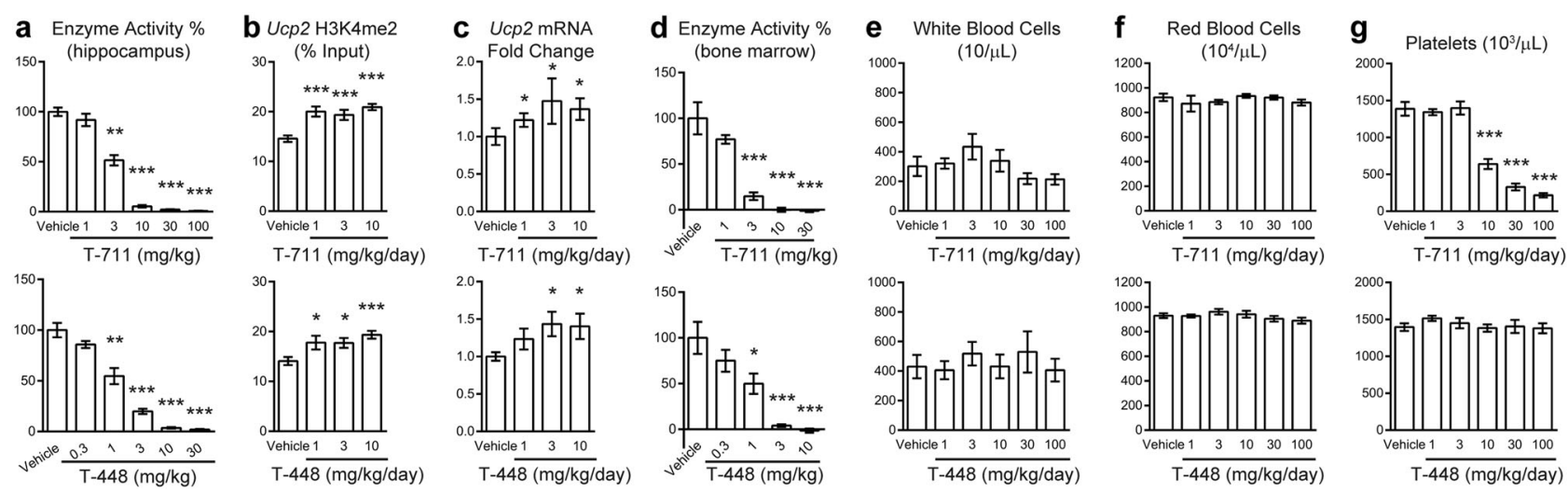

Fig. 4 T-448 increased brain H3K4me2 levels without hematological safety issue in mice. a LSD1 enzyme activities in the mouse hippocampus at $2 \mathrm{~h}$ after the single administration of compounds. $n=6$. One-tailed Shirley-Williams test vs vehicle-treated groups, ${ }^{* *} p<0.005,{ }^{* * *} p<$ 0.0005 . b, c H3K4me2 level at the upstream region of the Ucp2 gene in the mouse cortex (b) and the expression level of Ucp2 mRNA in the mouse hippocampus (c) 1 day after the 9-day repeated administration. $n=9$. One-tailed parametric Williams' (b) or Shirley-Williams test (c) vs vehicle-treated groups, ${ }^{*} p<0.025,{ }^{* * *} p<0.0005$. d LSD1 enzyme activities in the mouse bone marrow at $2 \mathrm{~h}$ after the single administration. $n=7$ (vehicle-treated groups) or 4 (compound-treated groups). One-tailed parametric Williams' test vs vehicle-treated groups, ${ }^{*} p<0.025$, ${ }^{* * *} p<0.0005$. e-g Numbers of white blood cells (e), red blood cells (f), and platelets $(\mathbf{g})$ in the whole blood 1 day after the 4-day repeated administration. $n=6$. Two-tailed Shirley-Williams (f, T-711) or parametric Williams' test (other Figures) vs vehicle-treated groups, ${ }^{* * *} p<0.0005$. All graphs indicate mean \pm SEM

We characterized in vivo hematological side effects of these compounds in ICR mice. The inhibition of LSD1 enzyme activity in hematopoietic lineage cells was determined by ex vivo enzyme assays using mouse bone marrow tissues. As shown in Fig. $4 \mathrm{~d}$, dose-dependent LSD1 inhibition by both T-711 and T-448 in the bone marrow was observed; T-711 at $10 \mathrm{mg} / \mathrm{kg}$ and T-448 at $3 \mathrm{mg} /$ $\mathrm{kg}$ fully inhibited LSD1 in the bone marrow. The effects of these compounds on blood cell counts (number of white blood cells, red blood cells, and platelets) in mouse peripheral blood were evaluated after a 4-day repeated administration. T-711 at $10 \mathrm{mg} /$ $\mathrm{kg} /$ day or higher caused a significant decrease in platelet numbers, while T-448 at up to $100 \mathrm{mg} / \mathrm{kg} /$ day did not cause any change in blood cell numbers (Fig. $4 \mathrm{e}-\mathrm{g}$ ). Two-week repeated treatment with $\mathrm{T}-711$ at $30 \mathrm{mg} / \mathrm{kg}$ caused a significant and remarkable reduction in both red blood cells and platelets, whereas two-week repeated treatment with T-448 at $100 \mathrm{mg} / \mathrm{kg}$ caused a significant, but limited $(14 \%)$ reduction in red blood cells without affecting white blood cells and platelets (Fig. S9). These results suggest the superior hematological safety profile of T-448 in ICR mice. 
T-448 restores learning function through modulating $\mathrm{H} 3 \mathrm{~K} 4$ methylation levels

The NMDAR plays a pivotal role in memory formation through promoting the expression of synaptic plasticity-related genes such as Bdnf, Arc, and Fos. Regulation of Bdnf, Arc, and Fos mRNA expression is also controlled by epigenetic mechanisms [1, 34-37]. Therefore, we used $\mathrm{Nr}^{\text {neo-l- }}$ (NMDA receptor NR1 subunit hypofunction: NR1-hypo) mice to assess the therapeutic potential of T-448. Administration of T-448 to NR1-hypo mice at $1 \mathrm{mg} / \mathrm{kg} /$ day (49\% inhibition in ICR mouse brain) and $10 \mathrm{mg} / \mathrm{kg} / \mathrm{day}(96 \%$ inhibition in ICR mouse brain) for 3 weeks dose-dependently increased the H3K4me2 levels around Bdnf, Arc, and Fos genes in the mouse hippocampus. The difference was significant at $10 \mathrm{mg} /$ kg/day (Fig. 5a). In contrast to H3K4me2 methylation, basal levels of Bdnf, Arc, and Fos gene mRNA were decreased in NR1-hypo mouse brains compared to wild-type mice (Fig. 5b). Treatment with T-448 did not change in Bdnf, Arc, and Fos mRNA levels in wild-type mouse brains, but exhibited a tendency to restore normal levels in NR1-hypo mouse brains at $10 \mathrm{mg} / \mathrm{kg} /$ day (Fig. $5 \mathrm{~b}$ ).

NR1-hypo mice exhibited learning deficits in correct choices in the water Y-maze test (Fig. 5c). Repeated administration of T-448 for 3 weeks resulted in partial but statistically significant and dosedependent rescue effects on the rate of correct choices in NR1hypo mice (Fig. $5 \mathrm{c}$ ). Taken together, these observations suggest that T-448 partially restored learning impaired by NMDAR hypofunction by increasing H3K4 methylation in the brain and consequently normalizing levels of neural plasticity-related gene expression.

\section{DISCUSSION}

Aberrant changes in gene expression may be associated with neurodevelopmental and neuropsychiatric disorders. In addition to genetic mutations, "gene-environment" interactions and epigenetic control is critical in brain function [38]. Organisms can adjust gene expression in response to intrinsic and environmental signals through epigenetic mechanisms, such as modification of chromatin structure and DNA methylation. Thus, dysfunction of epigenetic mechanisms may also underlie pathophysiology of CNS disorders [39, 40]. Among epigenetic pathways, $\mathrm{H} 3 \mathrm{~K} 4$ methylation is critically involved in various neural functions, such as learning and memory. For example, haploinsufficiency of the CHD8 (chromodomain helicase DNA-binding protein 8) gene, which encodes for a methylated histone reader, has been implicated as a cause of ASD [41, 42], suggesting that upregulation of $\mathrm{H} 3 \mathrm{~K} 4$ methylation may be a promising strategy to treat CNS disorders.

In this study, we discovered a novel LSD1 inhibitor, T-448, which increased H3K4me2 levels in the brain and improved learning in an NMDAR hypofunction mouse model without causing any change in blood cell numbers at the effective dose $(10 \mathrm{mg} / \mathrm{kg})$, suggesting that it has a wider safety margin against thrombocytopenia than do other known LSD1 inhibitors. This improved safety profile of T-448 would be due to its minimal impact on the LSD1-GFI1B complex and the lack of GFI1 mRNA induction in TF-1a cells. Co-crystal structural and LC/MS analyses revealed that T-367, the eutomer of T-711, produced a bulky FADadduct causing a steric clash with the GFI1B peptide. This steric interference may lead to the dissociation of the LSD1-GFI1B complex and GFI1 mRNA induction in TF-1a cells. In contrast, T448 produced a compact formyl-FAD adduct that could avoid the steric clash with cofactor GFI1B. As for the molecular mechanism, the production of formyl-FAD adduct would be mediated by the transient generation of full T-448-FAD adduct, because LC/MS analysis detected the time-dependent decrease of full T-448-FAD adduct $(m / z \quad 1,059)$ and the time-dependent increase of formylFAD $(m / z$ 814). In line with this hypothesis, SPR analysis showed the transient inhibition of the LSD1-GFI1B interaction by T-448.
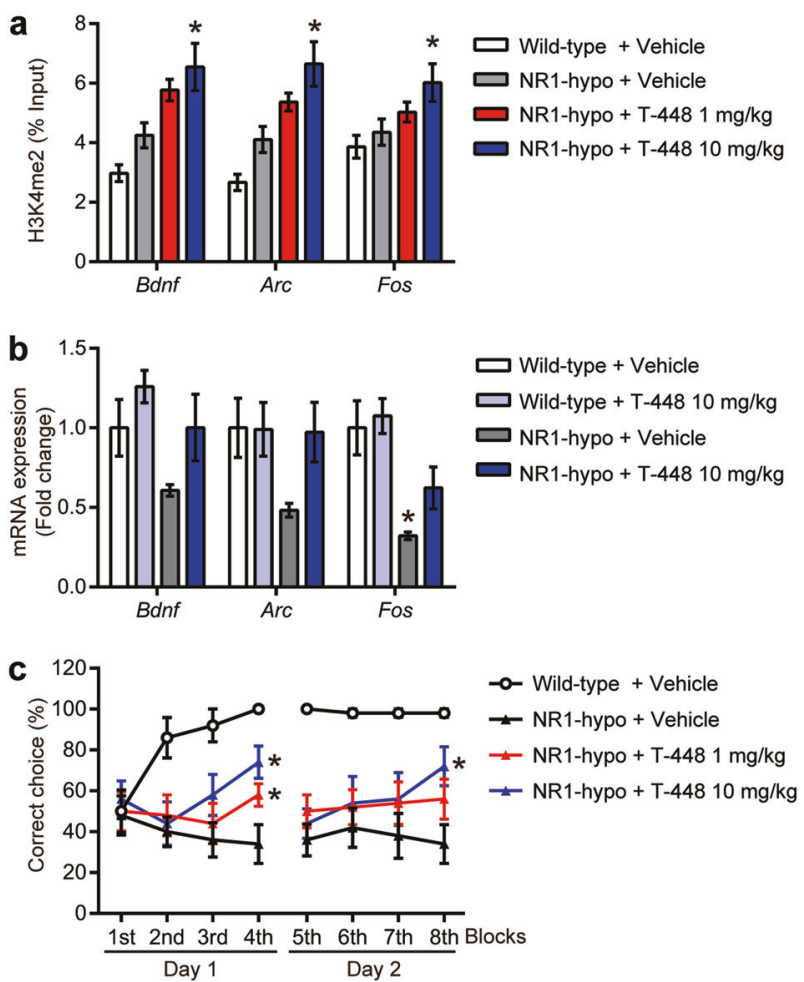

Fig. 5 T-448 treatment for 3 weeks increased brain H3K4me2 levels and restored learning function in NMDAR hypofunction mice. a H3K4me2 levels around Bdnf, Arc, and Fos genes in the wild-type and the NR1-hypo mouse hippocampus. Graphs indicate mean \pm SEM. $n=5$. One-tailed parametric Williams' test vs vehicle-treated NR1-hypo mouse group, ${ }^{*} p<0.025$. b mRNA expression levels of $B d n f, A r c$, and Fos genes in the wild type and the NR1-hypo mouse hippocampus. Graphs indicate mean \pm SEM. $n=4$. Welch's test vs vehicle-treated wild-type mouse group, ${ }^{*} p<0.05$. c Effects of T-448 on the learning behavior of NR1-hypo mice in water Y-maze test. Correct choice percentage was calculated in every block. Data are shown as mean + SEM. $n=10$. One-tailed parametric Williams' test vs vehicle-administered group at 4 th and 8th blocks, ${ }^{*} p<0.025$

Further studies are needed to understand these mechanisms of action in detail.

Platelet reduction after treatment with an LSD1 inhibitor that disrupts LSD1-complex was observed for at least 3 days after administration in mice [23]. If the transient disruption of the LSD1GFI1B interaction by T-448 caused platelet reduction, the blood cell examination performed 1 day after repeated administration for 4 days would reveal the changes in platelet numbers. Under the experimental conditions, T-711, but not T-448, reduced platelet numbers. This suggests that the transient disruption of the LSD1-GFI1B interaction by T-448 may not be associated with hematological adverse effects.

The discovery of T-448 enabled the in vivo characterization of therapeutic effects by the specific inhibition of LSD1 enzyme activity. NMDAR-dependent signal plays pivotal roles in the transcription of neural plasticity-related genes, such as Bdnf, Arc, and Fos [1]. In fact, reduced transcription level of Fos gene was observed in NR1-hypo mouse brain $[28,29]$. The increasing trend in H3K4me2 levels in NR1-hypo mice may be a compensatory mechanism for the impaired transcription. Repeated administration of T-448 at $10 \mathrm{mg} / \mathrm{kg}$ enhanced the H3K4me2 levels around $B d n f$, Arc, and Fos genes. These findings suggested that LSD1 inhibition restores cellular responsiveness to NMDAR-dependent signals via increasing the H3K4 methylation. Furthermore, T-448 at $10 \mathrm{mg} / \mathrm{kg}$ rescued the learning deficits in NR1-hypo mice. Inhibition of LSD1 enzyme activity could be an approach to treat 
T-448, a specific inhibitor of LSD1 enzyme activity, improves learning... $S$ Matsuda et al.

diseases associated with NMDAR hypofunction such as schizophrenia and ASD.

As discussed, epigenetic mechanisms may have pivotal roles in responding to environmental changes through the modification of gene expression. In other words, modulation of cellintrinsic signals or extracellular signals alone by the stimulation of signal transduction molecules or receptors would not be enough to fully modify disease pathophysiology with epigenetic dysfunction. Therefore, environmental factors should be carefully considered when assessing the therapeutic potential of T448. In addition to H3K4 demethylation, LSD1 is also involved in the demethylation of H3K9 [11] and non-histone proteins, such as DNMT1 [12] and p53 [43]. These molecules are also involved in neurocognitive function and associated with neurodevelopmental disorders [44, 45]. Thus, T-448 may produce its pharmacological effects through the modulation of both histone and non-histone substrates.

In summary, we discovered a novel LSD1 enzyme inhibitor, T448 , which has a minimal impact on the LSD1-GFI1B complex and on hematological liability in mice through the generation of a compact formyl-FAD adduct. T-448 increased brain H3K4 methylation levels and restored learning function in mice with NMDAR hypofunction. T-448-type LSD1 inhibitors with improved safety profiles can provide unique therapeutic opportunities for CNS disorders with epigenetic dysregulation.

\section{FUNDING AND DISCLOSURE}

This work was funded by Takeda Pharmaceutical Company Limited.

\section{ACKNOWLEDGEMENTS}

The authors thank Dr. Takayuki Niimura and Mr. Ryota Maeda for performing the pharmacological analysis, Mr. Yasushi Hattori and Dr. Shigemitsu Matsumoto for compound synthesis, Mr. Takashi Ito and Ms. Yumi Zama for protein purification, as well as Dr. Gyorgy Snell and Dr. Lane Scott for data collection and processing for crystal structure analysis. This work was funded by the Takeda Pharmaceutical Company Limited.

\section{AUTHOR CONTRIBUTIONS}

S.Matsuda and H.Kimura conceived and designed the experiments. S.Matsuda, R.B., H.O., S.Igaki, Y.K., S.Iwasaki, K.M., R.Hibino, H.Kamada, T.H., M.Iwatani, K.T., and R.Hara performed the experiments and analyzed the data. S.Morimoto, M.T., and M.Ito designed and synthesized T-448. S.Matsuda and H.Kimura wrote the manuscript.

\section{ADDITIONAL INFORMATION}

Supplementary Information accompanies this paper at (https://doi.org/10.1038/ s41386-018-0300-9).

Competing interests: The authors are present employees of the Takeda Pharmaceutical Company Limited or were employees of Takeda Pharmaceutical Company Limited while engaged in this research.

Publisher's note: Springer Nature remains neutral with regard to jurisdictional claims in published maps and institutional affiliations.

\section{REFERENCES}

1. Cortes-Mendoza J, Diaz de Leon-Guerrero S, Pedraza-Alva G, Perez-Martinez L. Shaping synaptic plasticity: the role of activity-mediated epigenetic regulation on gene transcription. Int J Dev Neurosci. 2013;31:359-69.

2. Guan JS, Xie H, Ding X. The role of epigenetic regulation in learning and memory. Exp Neurol. 2015;268:30-36.

3. Gupta S, Kim SY, Artis S, Molfese DL, Schumacher A, Sweatt JD, et al. Histone methylation regulates memory formation. J Neurosci. 2010;30:3589-99.

4. Huang HS, Matevossian A, Whittle C, Kim SY, Schumacher A, Baker SP, et al. Prefrontal dysfunction in schizophrenia involves mixed-lineage leukemia 1-regulated histone methylation at GABAergic gene promoters. J Neurosci. 2007a;27:11254-62.

5. Network, Pathway Analysis Subgroup of Psychiatric Genomics C. Psychiatric genome-wide association study analyses implicate neuronal, immune and histone pathways. Nat Neurosci. 2015;18:199-209.

6. Shulha HP, Cheung I, Whittle C, Wang J, Virgil D, Lin CL, et al. Epigenetic signatures of autism: trimethylated H3K4 landscapes in prefrontal neurons. Arch General Psychiatry. 2012;69:314-24.

7. Wynder C, Stalker L, Doughty ML. Role of H3K4 demethylases in complex neurodevelopmental diseases. Epigenomics. 2010;2:407-18.

8. Parkel S, Lopez-Atalaya JP, Barco A. Histone H3 lysine methylation in cognition and intellectual disability disorders. Learn Mem. 2013;20:570-9.

9. Vashishtha M, Ng CW, Yildirim F, Gipson TA, Kratter IH, Bodai L, et al. Targeting H3K4 trimethylation in Huntington disease. Proc Natl Acad Sci USA 2013;110: E3027-3036.

10. Schmitt ML, Ladwein Kl, Carlino L, Schulz-Fincke J, Willmann D, Metzger E, et al. Heterogeneous Antibody-based Activity Assay For Lysine Specific Demethylase 1 (LSD1) on a histone peptide substrate. J Biomol Screen. 2014;19:973-8.

11. Metzger E, Wissmann M, Yin N, Muller JM, Schneider R, Peters AH, et al. LSD1 demethylates repressive histone marks to promote androgen-receptordependent transcription. Nature. 2005;437:436-9.

12. Wang J, Hevi S, Kurash JK, Lei H, Gay F, Bajko J, et al. The lysine demethylase LSD1 (KDM1) is required for maintenance of global DNA methylation. Nat Genet. 2009;41:125-9.

13. Shi Y, Lan F, Matson C, Mulligan P, Whetstine JR, Cole PA, et al. Histone demethylation mediated by the nuclear amine oxidase homolog LSD1. Cell. 2004;119:941-53.

14. Stavropoulos P, Blobel G, Hoelz A. Crystal structure and mechanism of human lysine-specific demethylase-1. Nat Struct Mol Biol. 2006;13:626-32.

15. Yang M, Culhane JC, Szewczuk LM, Gocke CB, Brautigam CA, Tomchick DR, et al. Structural basis of histone demethylation by LSD1 revealed by suicide inactivation. Nat Struct Mol Biol. 2007;14:535-9.

16. Forneris F, Binda C, Dall'Aglio A, Fraaije MW, Battaglioli E, Mattevi A. A highly specific mechanism of histone H3-K4 recognition by histone demethylase LSD1. J Biol Chem. 2006;281:35289-95.

17. Wang $X$, Huang $B$, Suzuki T, Liu X, Zhan P. Medicinal chemistry insights in the discovery of novel LSD1 inhibitors. Epigenomics. 2015;7:1379-96.

18. Baron R, Binda C, Tortorici M, McCammon JA, Mattevi A. Molecular mimicry and ligand recognition in binding and catalysis by the histone demethylase LSD1CoREST complex. Structure. 2011;19:212-20.

19. Saleque S, Kim J, Rooke HM, Orkin SH. Epigenetic regulation of hematopoietic differentiation by Gfi- 1 and Gfi- $1 \mathrm{~b}$ is mediated by the cofactors CoREST and LSD1. Mol Cell. 2007;27:562-72.

20. Hock H, Hamblen MJ, Rooke HM, Schindler JW, Saleque S, Fujiwara Y, et al. Gfirestricts proliferation and preserves functional integrity of haematopoietic stem cells. Nature. 2004:431:1002-7.

21. Moroy $T$, Vassen $L$, Wilkes B, Khandanpour C. From cytopenia to leukemia: the role of Gfi1 and Gfi1b in blood formation. Blood. 2015;126:2561-9.

22. Sprussel A, Schulte JH, Weber S, Necke M, Handschke K, Thor T, et al. Lysinespecific demethylase 1 restricts hematopoietic progenitor proliferation and is essential for terminal differentiation. Leukemia. 2012;26:2039-51.

23. Ishikawa Y, Gamo K, Yabuki M, Takagi S, Toyoshima K, Nakayama K, et al. A novel LSD1 inhibitor T-3775440 disrupts GFI1B-containing complex leading to transdifferentiation and impaired growth of AML cells. Mol Cancer Ther 2017;16:273-84.

24. Mohammad HP, Smitheman KN, Kamat CD, Soong D, Federowicz KE, Van Aller GS, et al. A DNA hypomethylation signature predicts antitumor activity of LSD1 inhibitors in SCLC. Cancer Cell. 2015;28:57-69.

25. Rivers A, Vaitkus K, Ruiz MA, Ibanez V, Jagadeeswaran R, Kouznetsova T, et al. RN1 , a potent and selective lysine-specific demethylase 1 inhibitor, increases gamma-globin expression, $\mathrm{F}$ reticulocytes, and $\mathrm{F}$ cells in a sickle cell disease mouse model. Exp Hematol. 2015:43:546-53 e541-543.

26. Maiques-Diaz A, Spencer GJ, Lynch JT, Ciceri F, Williams EL, Amaral FMR, et al. Enhancer activation by pharmacologic displacement of LSD1 from GFI1 induces differentiation in acute myeloid leukemia. Cell Rep. 2018;22:3641-59.

27. Yamamoto R, Kawahara M, Ito S, Satoh J, Tatsumi G, Hishizawa M, et al. Selective dissociation between LSD1 and GFI1B by a LSD1 inhibitor NCD38 induces the activation of ERG super-enhancer in erythroleukemia cells. Oncotarget. 2018;9:21007-21.

28. Halene TB, Ehrlichman RS, Liang Y, Christian EP, Jonak GJ, Gur TL, et al. Assessment of NMDA receptor NR1 subunit hypofunction in mice as a model for schizophrenia. Genes Brain Behav. 2009;8:661-75.

29. Mohn AR, Gainetdinov RR, Caron MG, Koller BH. Mice with reduced NMDA receptor expression display behaviors related to schizophrenia. Cell. 1999;98:427-36. 
T-448, a specific inhibitor of LSD1 enzyme activity, improves learning...

S Matsuda et al.

30. Wesseling $\mathrm{H}$, Guest $\mathrm{PC}$, Lee $\mathrm{CM}$, Wong $\mathrm{EH}$, Rahmoune $\mathrm{H}$, Bahn S. Integrative proteomic analysis of the NMDA NR1 knockdown mouse model reveals effects on central and peripheral pathways associated with schizophrenia and autism spectrum disorders. Mol Autism. 2014;5:38.

31. Kunugi A, Tajima Y, Kuno H, Sogabe S, Kimura H. HBT1, a novel AMPA receptor potentiator with lower agonistic effect, avoided bell-shaped response in in vitro BDNF production. J Pharmacol Exp Ther. 2018;364:377-89.

32. Tomita N, Kajii S, Douglas RC, Tomita D, Imamura S, Tsuchida K, et al. Cyclopropanamine compound. Patent WO2013022047. 2013.

33. Rotili D, Mai A. Targeting histone demethylases: a new avenue for the fight against cancer. Genes Cancer. 2011;2:663-79.

34. Levenson JM, O'Riordan KJ, Brown KD, Trinh MA, Molfese DL, Sweatt JD. Regulation of histone acetylation during memory formation in the hippocampus. J Biol Chem. 2004;279:40545-59.

35. Boulle F, van den Hove DL, Jakob SB, Rutten BP, Hamon M, van Os J, et al. Epigenetic regulation of the BDNF gene: implications for psychiatric disorders. Mol Psychiatry. 2012;17:584-96.

36. Carter SD, Mifsud KR, Reul JM. Distinct epigenetic and gene expression changes in rat hippocampal neurons after Morris water maze training. Front Behav Neurosci. 2015;9:156.

37. Flavell SW, Greenberg ME. Signaling mechanisms linking neuronal activity to gene expression and plasticity of the nervous system. Annu Rev Neurosci. 2008;31:563-90.
38. Meaney MJ. Epigenetics and the biological definition of gene $x$ environment interactions. Child Dev. 2010;81:41-79.

39. Tordjman S, Somogyi E, Coulon N, Kermarrec S, Cohen D, Bronsard G, et al. Gene $x$ Environment interactions in autism spectrum disorders: role of epigenetic mechanisms. Front Psychiatry. 2014;5:53.

40. van Vliet J, Oates NA, Whitelaw E. Epigenetic mechanisms in the context of complex diseases. Cell Mol life Sci. 2007;64:1531-8.

41. Barnard RA, Pomaville MB, O'Roak BJ. Mutations and modeling of the chromatin remodeler CHD8 define an emerging autism etiology. Front Neurosci. 2015; 9:477.

42. Katayama $Y$, Nishiyama M, Shoji H, Ohkawa $Y$, Kawamura A, Sato T, et al. CHD8 haploinsufficiency results in autistic-like phenotypes in mice. Nature. 2016;537:675-9.

43. Huang J, Sengupta R, Espejo AB, Lee MG, Dorsey JA, Richter M, et al. p53 is regulated by the lysine demethylase LSD1. Nature. 2007b;449:105-8.

44. Irwin RE, Pentieva K, Cassidy T, Lees-Murdock DJ, McLaughlin M, Prasad G, et al. The interplay between DNA methylation, folate and neurocognitive development. Epigenomics. 2016;8:863-79.

45. Papiol S, Arias B, Barrantes-Vidal N, Guitart M, Salgado P, Catalan R, et al. Analysis of polymorphisms at the tumor suppressor genep53 (TP53) in contributing to the risk for schizophrenia and its associated neurocognitive deficits. Neurosci Lett. 2004;363:78-80. 